\title{
Efficacy and tolerability of the
} investigational topical cream SD-101 (6\% allantoin) in patients with epidermolysis bullosa: a phase 3, randomized, doubleblind, vehicle-controlled trial (ESSENCE study)

Amy S. Paller ${ }^{1 *}$, John Browning ${ }^{2}$, Milos Nikolic ${ }^{3}$, Christine Bodemer ${ }^{4}$, Dedee F. Murrell ${ }^{5}$, Willistine Lenon ${ }^{6}$, Eva Krusinska ${ }^{7}$, Allen Reha ${ }^{7}$, Hjalmar Lagast ${ }^{7}$, Jay A. Barth ${ }^{7}$ on behalf of the ESSENCE Study Group

\begin{abstract}
Background: Epidermolysis bullosa (EB) is a rare genetic disorder that manifests as blistering and/or skin erosion. There is no approved treatment for EB; current standard of care consists of wound and pain management. SD-101 $6 \%$ is a topical cream containing $6 \%$ allantoin that was developed for treating skin lesions in patients with EB. The aim of this phase 3, multicenter, randomized, double-blind, vehicle-controlled study was to assess the efficacy and safety of SD-101 6\% cream versus vehicle (0\% allantoin) on lesions in patients with EB.

Methods: Eligible patients were $\geq 1$ month old, had a diagnosis of EB (simplex, recessive dystrophic, or intermediate junctional) and a target wound $10-50 \mathrm{~cm}^{2}$ in size that was present for $\geq 21$ days. Patients were randomly assigned to SD-101 6\% cream or vehicle, which was applied topically once a day to the entire body for 3 months. Primary efficacy endpoints were time to complete target wound closure within 3 months and the proportion of patients who experienced complete target wound closure within 3 months. Post hoc subgroup analyses were conducted by patient age and in those with body surface area index of total body wound burden $\geq 5 \%$ at baseline.

(Continued on next page)
\end{abstract}

*Correspondence: apaller@northwestern.edu

Eva Krusinska: Author working as a consultant under the contract of

Pharmaland Consulting Group

${ }^{1}$ Northwestern University Feinberg School of Medicine, Chicago, IL, USA

Full list of author information is available at the end of the article

(c) The Author(s). 2020 Open Access This article is licensed under a Creative Commons Attribution 4.0 International License, which permits use, sharing, adaptation, distribution and reproduction in any medium or format, as long as you give appropriate credit to the original author(s) and the source, provide a link to the Creative Commons licence, and indicate if changes were made. The images or other third party material in this article are included in the article's Creative Commons licence, unless indicated otherwise in a credit line to the material. If material is not included in the article's Creative Commons licence and your intended use is not permitted by statutory regulation or exceeds the permitted use, you will need to obtain permission directly from the copyright holder. To view a copy of this licence, visit http://creativecommons.org/licenses/by/4.0/ The Creative Commons Public Domain Dedication waiver (http://creativecommons.org/publicdomain/zero/1.0/) applies to the data made available in this article, unless otherwise stated in a credit line to the data. 
(Continued from previous page)

Results: In total, 169 patients were enrolled and randomly assigned to SD-101 6\% ( $n=82)$ or vehicle $(n=87)$. Baseline demographics and disease characteristics were similar between treatment groups. There were no statistically significant differences between treatment groups in time to target wound closure (hazard ratio, 1.004; 95\% confidence interval [Cl] 0.651, 1.549; $P=0.985)$ or proportion of patients with complete target wound closure within 3 months (odds ratio [95\% Cl], 0.733 [0.365, 1.474]; nominal $P=0.390$ ). A positive trend toward faster wound closure with SD-101 6\% versus vehicle was observed in patients aged 2 to $<12$ years and those with total body wound burden $\geq 5 \%$ at baseline. SD-101 6\% cream was well tolerated.

Conclusions: SD-101 6\% cream for treatment of EB-associated lesions was not more effective than vehicle in shortening the time to complete target wound closure or achieving complete target wound closure within 3 months.

Trial registration: ClinicalTrials.gov, NCT02384460; Date of trial registration, February 13, 2015; First participant enrolled, March 11, 2015.

Keywords: Epidermolysis bullosa, Efficacy, Safety, SD-101, Wound closure, Allantoin

\section{Introduction}

Epidermolysis bullosa (EB) comprises a group of rare clinically and genetically heterogeneous disorders [1, 2], characterized by fragile skin and mucous membranes, causing blistering or erosions in response to minimal or no trauma [2]. The cutaneous and extracutaneous manifestations of EB can cause serious complications and significant morbidity, and in some cases, premature death [1]. Wound infection can lead to life-threatening sepsis, and in some EB subtypes, there is an increased risk for aggressive squamous cell carcinomas [1-4]. The chronic pain associated with $\mathrm{EB}$, the hardship placed on caregivers, and the high risk for complications places a considerable psychosocial burden on both patients and their families [5-8].

The incidence of EB is estimated to be approximately 1 in 50,000 live births [9]. Most patients with EB are diagnosed with 1 of 3 types based on the ultrastructural level of skin cleavage: simplex (70\%), dystrophic (20\%), or junctional $(10 \%)[2,10,11]$. Further subclassifications are based on the affected gene and the extent of skin and mucosal disease. Diagnosis often occurs in the neonatal period, although lesions may not appear in some individuals until adolescence or later, delaying an accurate diagnosis until adulthood [6].

Despite considerable research to advance the understanding of EB pathophysiology, no treatments have been approved by regulatory authorities to date $[3,12]$. Current standard of care consists of bandaging and cleaning open wounds to prevent infection, pain management, and symptomatic treatment of complications $[1,2,13]$. Consequently, there is a significant unmet need for effective medical therapies.

SD-101 6\% is a topical cream containing 6\% allantoin that was designed for the treatment of wounds and other skin lesions in patients with EB $[14,15]$. The efficacy and safety of SD-101 6\% was previously investigated in a phase $2 \mathrm{~b}$, multicenter, randomized, double-blind, vehicle-controlled
(0\% allantoin), dose-ranging study (ClinicalTrials.gov: NCT02014376) in patients with EB aged $\geq 6$ months $(N=$ 48) [14]. Results showed that, compared with vehicle, treatment with SD-101 6\% resulted in a numerically higher rate of wound closure at 1 month $(67 \%$ vs $41 \%, P=0.165)$ and a significantly higher rate of wound closure at 2 months $(82 \%$ vs $41 \%, P=0.04$ ) [14]. Patients in the SD-101 $6 \%$ group also achieved faster target wound closure and had a greater reduction in lesional body surface area (BSA) than patients in the vehicle group. SD-101 6\% was well tolerated and had a safety profile similar to that of vehicle. Herein, we report the results of the 3-month, double-blind, vehicle-controlled, phase 3 study (ESSENCE), which compared the efficacy and safety of SD-101 6\% with that of vehicle in a large population of patients with EB.

\section{Methods}

\section{Study design and treatment}

ESSENCE (ClinicalTrials.gov NCT02384460) was a multicenter, randomized, double-blind, vehicle-controlled, phase 3 trial designed to assess the efficacy and safety of SD-101 6\% versus vehicle (SD-101 0\%) in patients with simplex, recessive dystrophic, or intermediate junctional EB (Fig. 1) [16]. The study was conducted between March 2015 and July 2017 in 13 countries. Patients were randomly assigned 1:1 to receive SD-101 6\% or vehicle using an interactive web response system. Both SD-101 and vehicle were applied topically once daily to the entire body (including non-wounded areas) as a thin layer for 90 days. SD-101 6\% is a topical cream containing 6\% allantoin in an oil-in-water emulsion. The vehicle consisted of the same cream formulation as SD-101 but excluded allantoin. SD-101 and the vehicle contained the following excipients: beeswax, butylated hydroxytoluene, cetyl alcohol, citric acid, cod liver oil, lanolin oil, methylparaben, propylene glycol, propylparaben, sodium lauryl sulfate, stearyl alcohol, tetrasodium ethylenediaminetetraacetic 


\section{Daily whole-body application of cream}

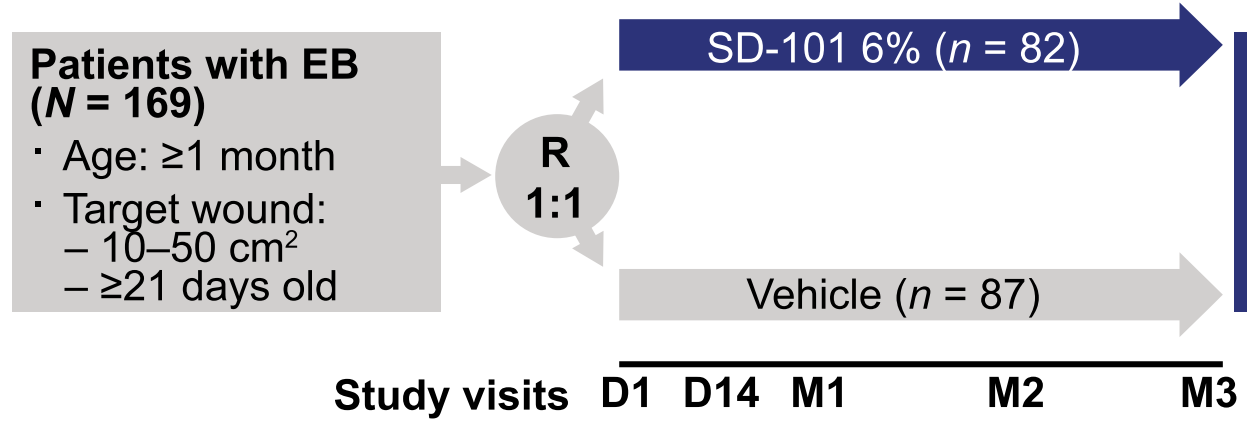

Fig. 1 ESSENCE study design. EB epidermolysis bullosa; $D$ day; $M$ month; $R$ randomization

acid, and purified water. Patient visits occurred at baseline, Day 14, Month 1, Month 2, and Month 3; those who completed the study were eligible to enroll in an open label extension study.

\section{Patients}

Patients were eligible for study participation if they were at least 1 month old and had a diagnosis of simplex, recessive dystrophic, or intermediate junctional EB based on a skin biopsy with immunofluorescence mapping, genetic testing, or, when genetic testing was not available, medical records. In these cases, specific features of EB that allow a diagnosis to be made included hyperkeratosis of the palms and soles for EB simplex, pseudosyndactyly with esophageal strictures for recessive dystrophic $\mathrm{EB}$, and enamel defects and facial granulation tissue for intermediate junctional EB [17]. Patients must have had a target wound between 10 and $50 \mathrm{~cm}^{2}$ in size that was present for at least 21 days based on patient history. Screening and baseline visits could be combined if patients were eligible. Patients were excluded from the study if they had clinical evidence of local infection in the target wound, used any investigational drug or systemic or topical steroidal therapy within 30 days before enrollment (inhaled steroids and ophthalmic drops containing steroids were allowed), used immunotherapy or cytotoxic chemotherapy within 60 days before enrollment, used systemic antibiotics within 7 days before enrollment, had a current or past malignancy, or had arterial or venous disorder resulting in ulcerated lesions.

\section{Endpoints}

The primary endpoints of the study were selected in conjunction with the US Food and Drug Administration advisors, based on previous wound-healing studies. These were time to complete target wound closure within 3 months and the proportion of patients with target wound closure within 3 months. Key secondary endpoints were the proportion of patients with target wound closure at Months 1 and 2, the change in BSA index (BSAi) of lesional skin and total body wound burden at Month 3, change from baseline in patient-reported itching, and change from baseline in patient-reported pain.

\section{Assessments}

For each patient, a target wound was selected at baseline by the investigator and measured with the SilhouetteStar ${ }^{\mathrm{rm}}$ device (ARANZ Medical, Christchurch, New Zealand), a portable, quantitative wound imaging, measurement, and documentation system. At each study visit, the Silhouet$\operatorname{teStar}^{\text {TM }}$ device was used to assess complete target wound closure, defined as skin re-epithelialization without drainage.

Lesional skin, defined as skin with blisters, erosions, ulcerations, scabbing, bullae, and eschars, as well as areas that were weeping, sloughing, oozing, crusted, and/or denuded, was assessed at each visit by the same study physician. Areas of erythema and hyperpigmentation or hypopigmentation were not considered active lesions. To calculate the BSAi of lesional skin, the percentage (ranging from 0 to 100\%) of the affected area was recorded for each defined body region (head/neck, upper limbs, trunk [including groin], and lower limbs), multiplied by a weighting factor, then summed for all body regions. The BSAi of total wound burden was calculated as the percentage of total body coverage of open wounds, defined as an open area on the skin (epidermal covering is disrupted).

Itching was assessed using the Itch Man Pruritus Assessment Tool at each study visit [18]. For patients 6 years of age and older, responses were recorded in patient diaries; for patients $\leq 5$ years of age, caretakers provided responses regarding patient itching. Itching was scored on a scale from 0 (comfortable, no itch) to 4 (itches most terribly; impossible to sit still or concentrate).

Pain was assessed using the Face, Legs, Activity, Cry, Consolability (FLACC) scale for patients aged 1 month 
to 3 years and the Wong-Baker FACES ${ }^{\circ}$ Pain Scale for patients aged $\geq 4$ years $[19,20]$. Each of the 5 categories in the FLACC scale is scored from 0 to 2, with a cumulative score ranging from 0 to 10 . The Wong-Baker FACES $^{\circ}$ Pain Scale also ranges from 0 to 10 . For both scales, higher scores indicate greater pain.

Safety was assessed via monitoring the number and incidence of adverse events (AEs), body temperature, and physical examinations. A nontreatment-emergent $\mathrm{AE}$ was defined as an $\mathrm{AE}$ that originated before the first application of study drug and did not change in severity or relationship to treatment on or after the date of the first application of study drug. A treatment-emergent $\mathrm{AE}$ (TEAE) was defined as an AE that occurred on or after the first date of application of study drug. If the severity of the $\mathrm{AE}$ or its relationship to study drug changed on or after the first application of study drug, but before the end of the study, then that AE was considered a TEAE. Relationship of AEs to treatment was assessed by the investigator. No clinical laboratory tests were performed.

\section{Statistics}

The sample size estimation assumed that $35 \%$ of vehicletreated patients and $60 \%$ of SD-101-treated patients would experience complete closure of their target wound at or before the 3-month follow-up visit, which corresponds to a hazard ratio of 2.127 assuming exponential hazards over time. Using these assumptions and a 1sided overall alpha of 0.025 , it was estimated that approximately 150 patients would be required (75 in each group) to provide at least $86 \%$ power to observe a difference in the time to complete target wound closure between treatment groups. Efficacy was assessed in all patients randomly assigned to a study treatment (intentto-treat [ITT] population); safety was assessed in all patients who applied or were administered the study medication at least once (safety population).

The two primary efficacy endpoints were tested hierarchically; attainment of statistical significance for the first primary endpoint was required for continued formal testing. The first primary endpoint, time to complete target wound closure, was measured from the date of the first administration of the study drug to the date of target wound closure. Target wounds were considered closed at all time points following the first wound closure, even if it reopened. Both primary efficacy endpoints used a Cox proportional hazards model to compare treatment groups, with baseline target wound size, target wound age, and EB type as covariates. For time-towound-closure analyses, data were right censored at 3 months or time of withdrawal from the study.

Formal statistical testing of the second primary endpoint, the proportion of patients with target wound closure within 3 months, was performed by comparing treatment groups using the logistic regression model with baseline target wound size, target wound age, and EB type as covariates; missing values were imputed using multiple imputation methodology (missing at random).

The proportion of patients experiencing complete closure of their target wound (at 2 months or 1 month) was assessed using the same approach as the primary analysis. Changes from baseline in BSAi of lesional skin and total body wound burden were analyzed using a mixed-model repeated measures approach, including treatment, baseline BSAi, EB type, visit, and visittreatment interaction as fixed effects. Any improvements from baseline in itching and pain were compared between treatment groups using a logistic regression model with covariates of baseline score and EB type.

Exploratory subgroup analyses were conducted to assess the consistency of treatment effects on the primary efficacy endpoints. Planned subgroup analyses include response by age at baseline ( 28 days to $<2$ years; 2 years to $<12$ years; 12 years to $<18$ years; $\geq 18$ years). Post hoc analyses were conducted for patients with BSAi of total body wound burden $\geq 5$ and $<5 \%$ at baseline, target wound age of $\leq 30$ or $>30$ days, and by EB subtype. Hazard ratios, 95\% confidence intervals (CIs), and nominal $P$ values were calculated for the subgroup analyses.

Incidence of skin infections in each treatment group was compared using Fisher's exact test and summarized by treatment group (post hoc). Skin infections were defined as the following preferred terms: skin infection, wound infection, staphylococcal skin infection, skin bacterial infection, wound infection staphylococcal, folliculitis, wound infection bacterial, cellulitis, cellulitis staphylococcal, impetigo, infected skin ulcer, postoperative wound infection, and rash pustular.

\section{Results \\ Patients}

In total, 169 patients were enrolled from 13 countries worldwide (Australia, $n=11$; Austria, $n=1$; France, $n=19$; Germany, $n=16$; Israel, $n=6$; Italy, $n=8$; Lithuania, $n=4$; Netherlands, $n=3$; Poland, $n=8$; Serbia, $n=16$; Spain, $n=12$; United Kingdom, $n=5$; United States, $n=60$ ). Eighty-two patients were randomly assigned to receive SD-101 6\% and 87 were randomly assigned to receive vehicle (Fig. 2). Fourteen patients prematurely discontinued and 155 completed the study. Reasons for premature discontinuations included AEs (SD-101 $6 \% n=5$, vehicle $n=$ 2 ), withdrawal by subject (SD-101 $6 \% n=0$, vehicle $n=3$ ), and other (SD-101 $6 \% n=2$, vehicle $n=2)$.

There were no notable differences in baseline characteristics between treatment groups (Table 1). Mean (range) age was $13.9(0.2,67.0)$ years, with approximately half (49.7\%) of patients falling between the ages of 2 to $<12$ years of age. The SD-101 6\% group had more males 


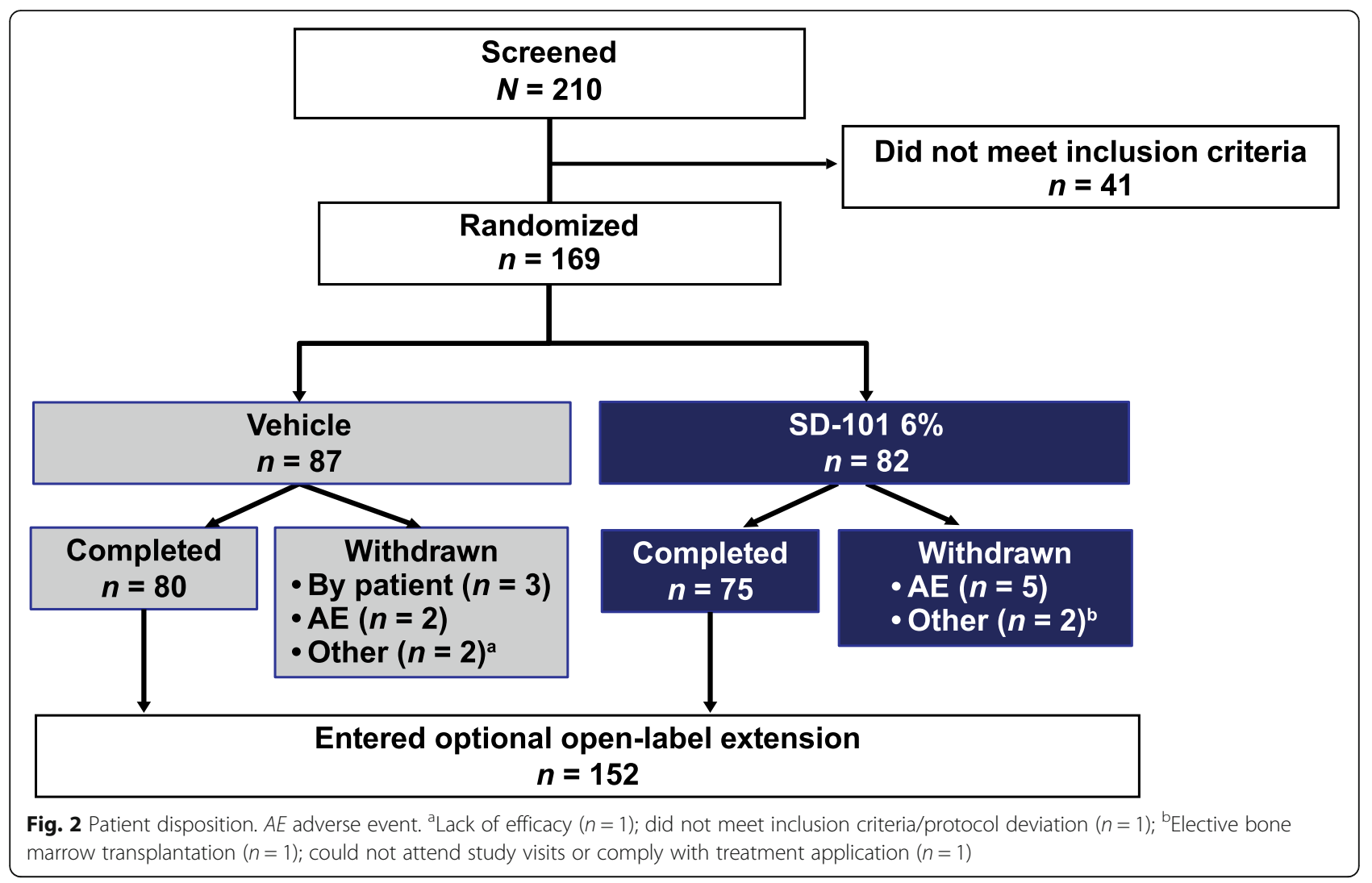

(59.8\%) and the vehicle group had more females (55.2\%). Most patients had recessive dystrophic EB.

\section{Efficacy results in the overall study population}

There were no statistically significant differences between the SD-101 6\% and vehicle groups on the primary efficacy endpoints of time to target wound closure (hazard ratio $[\mathrm{HR}] 1.00 ; 95 \% \mathrm{CI} 0.65,1.55 ; P=0.985$; Fig. 3a) and the proportion of patients with complete target wound closure within 3 months ( $49.4 \%$ vs $53.6 \%$ in the SD-101 6\% and vehicle groups, respectively; OR [95\% CI], 0.73 [0.37, 1.47]; nominal $P=0.390$; Table 2 and Fig. $3 \mathrm{~b})$. There were also no significant differences between the treatment groups on the key secondary endpoints, including the proportion of patients with complete target wound closure at Months 1 and 2, changes in BSAi of lesional skin or total body wound burden at Month 3 , and changes in itching or pain assessments at Day 7 (Table 2) and Month 3 (data not shown).

\section{Subgroup analyses for target wound closure}

In the subgroup of patients aged 2 to $<12$ years, there were no statistically significant differences in favor of the SD-101 6\% group compared with the vehicle-control group for the primary efficacy endpoints and most key secondary efficacy endpoints. There was a trend toward faster wound closure with SD-101 6\% versus vehicle within 3 months (HR 1.8; 95\% CI $0.9,3.5$; nominal $P=$ 0.085). The proportion of patients with complete target wound closure within 3 months was similar between treatment arms (55.6\% versus $46.7 \%$; OR [95\% CI], 1.3 $[0.5,3.6]$; nominal $P=0.658)$. However, the proportion of patients with complete target wound closure was significantly higher for the SD-101 6\% group compared with the vehicle group at Week $2(19.4 \%$ versus $2.2 \%$; OR [95\% CI], 10.9 [1.2, 99.8]; $P=0.034$ ) and Month 1 (41.7\% versus $20.0 \%$; OR [95\% CI], 3.4 [1.1, 10.8]; nominal $P=0.036$ ).

In the subgroup of patients with $\geq 5 \%$ BSAi of total wound burden at baseline, no significant differences between treatment groups were observed for the primary efficacy endpoints and most key secondary efficacy endpoints. There was a trend toward faster wound closure with SD-101 6\% versus vehicle within 3 months (HR 1.6; 95\% CI $0.9,2.8$; nominal $P=0.128$ ). Significantly more patients in the SD-101 6\% group had complete target wound closure within 1 month than those in the vehicle group (35.4\% versus 11.3\%; odds ratio [95\% CI], 3.995 $[1.319,12.104]$, nominal $P=0.014)$.

Although differences between treatment groups for the primary efficacy endpoints and most key secondary efficacy endpoints were also assessed by target wound age ( $\leq 30$ or $>30$ days) and EB subtype, these analyses did not provide significant findings (data not shown). 
Table 1 Baseline demographics and characteristics (ITT population)

\begin{tabular}{|c|c|c|c|}
\hline & $\begin{array}{l}\text { SD-101 6\% } \\
n=82\end{array}$ & $\begin{array}{l}\text { Vehicle } \\
n=87\end{array}$ & $\begin{array}{l}\text { Total } \\
N=169\end{array}$ \\
\hline Male, n (\%) & $49(59.8)$ & $39(44.8)$ & $88(52.1)$ \\
\hline \multicolumn{4}{|l|}{ Age, years } \\
\hline Mean \pm SD & $13.8 \pm 13.2$ & $13.9 \pm 13.1$ & $13.9 \pm 13.1$ \\
\hline Median & 9.0 & 10.5 & 10.0 \\
\hline Min, $\max$ & $0.2,67.0$ & $0.2,64.0$ & $0.2,67.0$ \\
\hline \multicolumn{4}{|l|}{ Age group, n (\%) } \\
\hline 1 month to $<2$ years & $5(6.1)$ & $6(6.9)$ & $11(6.5)$ \\
\hline 2 years to $<12$ years & $37(45.1)$ & $47(54.0)$ & $84(49.7)$ \\
\hline 12 years to $<18$ years & $19(23.2)$ & $12(13.8)$ & $31(18.3)$ \\
\hline$\geq 18$ years & $21(25.6)$ & $22(25.3)$ & $43(25.4)$ \\
\hline \multicolumn{4}{|l|}{ Race, n (\%) } \\
\hline White & $69(84.1)$ & $72(82.8)$ & $141(83.4)$ \\
\hline Black or African American & $5(6.1)$ & $3(3.4)$ & $8(4.7)$ \\
\hline Asian & $4(4.9)$ & $8(9.2)$ & $12(7.1)$ \\
\hline Other/not reported & $4(4.9)$ & $4(4.6)$ & $8(4.7)$ \\
\hline \multicolumn{4}{|l|}{ Epidermolysis bullosa type, n (\%) } \\
\hline Simplex & $10(12.2)$ & $8(9.2)$ & $18(10.7)$ \\
\hline Recessive dystrophic & $57(69.5)$ & $62(71.3)$ & $119(70.4)$ \\
\hline Intermediate junctional & 15 (18.3) & $17(19.5)$ & $32(18.9)$ \\
\hline \multicolumn{4}{|l|}{ Diagnosis, n (\%) } \\
\hline Genetic testing & $36(43.9)$ & $35(40.2)$ & $71(42.0)$ \\
\hline Immunomapping & $13(15.9)$ & $9(10.3)$ & $22(13.0)$ \\
\hline Medical records & $33(40.2)$ & $43(49.4)$ & $76(45.0)$ \\
\hline Target wound size, $\mathrm{cm}^{2}$, mean \pm SD & $18.8 \pm 12.1$ & $22.0 \pm 31.7$ & $20.4 \pm 24.2$ \\
\hline Target wound age, days, mean \pm SD & $406.5 \pm 913.8$ & $521.0 \pm 1832.0$ & $465.4 \pm 1457.4$ \\
\hline BSAi of lesional skin, $\%$, mean \pm SD & $25.8 \pm 19.4$ & $24.4 \pm 19.3$ & $25.1 \pm 19.3$ \\
\hline BSAi of total body wound burden, $\%$, mean \pm SD & $12.2 \pm 12.6$ & $10.5 \pm 9.1$ & $11.3 \pm 11.0$ \\
\hline Baseline itching score ${ }^{a}$, mean $\pm S D$ & $1.8 \pm 1.21$ & $2.0 \pm 1.14$ & NC \\
\hline Baseline pain score ${ }^{\mathrm{b}}$, mean $\pm S D$ & $3.1 \pm 2.57$ & $3.5 \pm 2.88$ & NC \\
\hline
\end{tabular}

$B S A i$ body surface area index; ITT intent-to-treat; $N C$ not calculated; SD standard deviation

${ }^{\text {a }}$ Scale from 0 to 4

${ }^{\text {b }}$ Scale from 0 to 10

\section{Safety}

SD-101 6\% was well tolerated with a safety profile similar to that of vehicle (Table 3). Overall, pruritus that occurred or worsened after the first administration of either treatment was the most common TEAE, occurring in 9 patients in the SD-101 6\% arm and 8 patients in the vehicle arm (Table 3). Skin infections of interest, which comprised 13 skin infection-related preferred terms, were significantly less frequent in the SD-101 6\% arm compared with the vehicle arm (18\% versus $33.3 \% ; P=0.026$ ) (Table 3 ).

AEs leading to study discontinuation and serious AEs were uncommon in both treatment groups (Table 3). AEs considered by the investigator to be treatment-related were similar in both treatment groups (Table 4). Pruritus was the most common treatment-related $\mathrm{AE}$, occurring in 6 patients in the SD-101 6\% arm and 5 in the vehicle arm.

One death was reported in the vehicle treatment arm. The patient was a 10-year-old male with intermediate junctional EB who was withdrawn from the study by his father on day 28. On day 38 , the child was diagnosed with influenza, which was ongoing on day 62 , when he died from cardiopulmonary failure. The death was considered by the investigator to be unrelated to treatment.

\section{Discussion}

EB is a devastating inherited disorder for which the greatest unmet treatment needs include effective wound healing and prevention, and control of pain and itch [21]. SD-101 

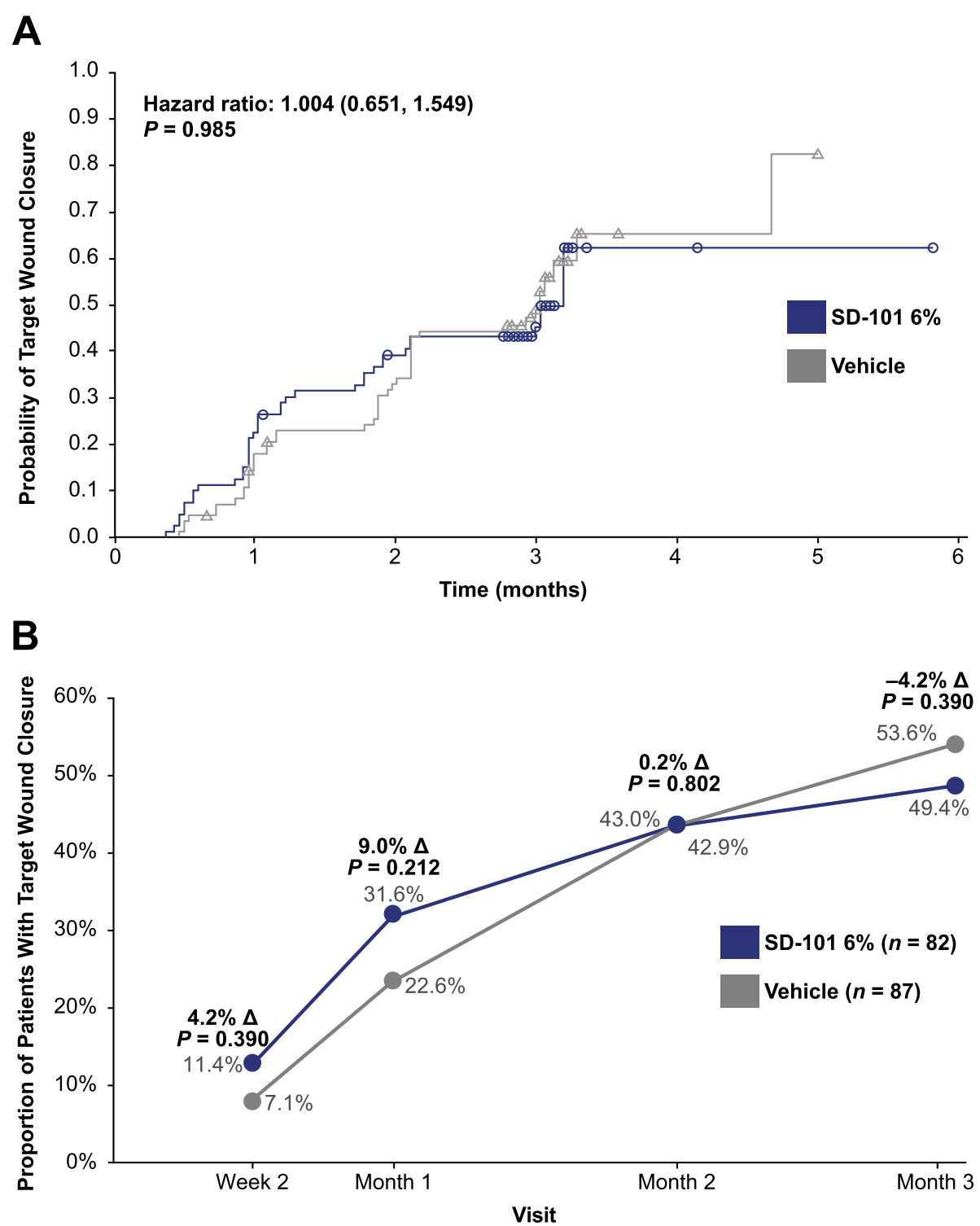

Fig. 3 (a) Time to target wound closure and (b) proportion of patients with target wound closure by visit ${ }^{\mathrm{a}}$. ITT intent-to-treat. ${ }^{\mathrm{a}}$ ITT population $(N=169)$. Patients were censored if they did not have a response within 3 months or withdrew early before the confirmation of their target wound closing. The Kaplan-Meier curves exceed the time limit of 3 months because the study duration for a fraction of patients was more than 3 months (nominal Month 3 visit could have occurred at Month 5)

6\% was developed as a topical therapy for the treatment of EB and is designed to deliver a high concentration $(6 \%)$ of allantoin to lesional skin in a highly stable, soluble form. In preclinical studies, allantoin was shown to have multiple wound-healing effects, including anti-inflammatory and antimicrobial activity as well as promoting tissue formation and differentiation, specifically in stimulating collagen deposition and epithelialization [22, 23]. Allantoin is excreted in maggot urine, which may explain the effectiveness of maggot therapy in wound healing [24]. In humans, allantoin is produced in the amniotic membranes only, which themselves have been used to treat wounds of patients with recessive dystrophic EB [25]. Evidence for the clinical benefit of SD-101 6\% was observed in the phase $2 b$ study, with a numerically higher rate of wound closure after 1 month compared with vehicle [14]. Data from this study provided the basis for the present phase 3 trial, which assessed the efficacy and safety of SD-101 6\% over 3-months' duration in a large, heterogeneous EB patient population with a broad range of demographic and disease characteristics. However, no statistically significant differences were observed in primary or key secondary efficacy endpoints in the intent-totreat population $(n=169)$. These observations are in contrast to results from the phase $2 \mathrm{~b}$ trial, in which SD-101 6\% 
Table 2 Comparison of primary endpoints and key secondary endpoints (ITT population)

\begin{tabular}{|c|c|c|c|}
\hline & $\begin{array}{l}\text { SD-101 6\% } \\
n=82\end{array}$ & $\begin{array}{l}\text { Vehicle } \\
n=87\end{array}$ & $\begin{array}{l}\text { Nominal } \\
P \text { value }\end{array}$ \\
\hline \multicolumn{4}{|l|}{ Primary endpoints } \\
\hline Time to complete target wound closure within 3 months, mean (SD), day & $\begin{array}{l}(n=39) \\
41.6(25.5)\end{array}$ & $\begin{array}{l}(n=45) \\
53.6(28.6)\end{array}$ & 0.985 \\
\hline Complete closure of target wound within 3 months, response rate & $\begin{array}{l}(n=79) \\
49.4 \%\end{array}$ & $\begin{array}{l}(n=84) \\
53.6 \%\end{array}$ & 0.390 \\
\hline \multicolumn{4}{|l|}{ Secondary endpoints } \\
\hline Complete closure of target wound within 2 months, response rate & $\begin{array}{l}(n=79) \\
43.0 \%\end{array}$ & $\begin{array}{l}(n=84) \\
42.9 \%\end{array}$ & 0.802 \\
\hline Complete closure of target wound within 1 month, response rate & $\begin{array}{l}(n=79) \\
31.6 \%\end{array}$ & $\begin{array}{l}(n=84) \\
22.6 \%\end{array}$ & 0.212 \\
\hline Change in BSAi of lesional skin at Month 3, \% & $(n=75)$ & $(n=78)$ & \\
\hline Mean (SEM) & $-4.4(1.05)$ & $-5.0(1.53)$ & 0.706 \\
\hline Median (range) & $-2.7(-37.4,18.0)$ & $-3.0(-69.2,26.5)$ & \\
\hline Change in BSAi of total body wound burden at Month 3, \% & $(n=75)$ & $(n=79)$ & \\
\hline Mean (SEM) & $-3.5(0.94)$ & $-2.3(0.70)$ & 0.900 \\
\hline Median (range) & $-1.5(-42.6,12.5)$ & $-1.4(-24.1,17.0)$ & \\
\hline Change in itching score at Day 7 & $(n=77)$ & $(n=79)$ & \\
\hline Mean (SEM) & $-0.5(0.15)$ & $-0.3(0.14)$ & 0.262 \\
\hline Median (range) & $0.0(-4,2)$ & $0.0(-4,3)$ & \\
\hline Change in pain score at Day 7 & $(n=77)$ & $(n=80)$ & \\
\hline Mean (SEM) & $-0.3(0.29)$ & $-0.6(0.34)$ & 0.098 \\
\hline Median (range) & $0.0(-6,10)$ & $0.0(-8,10)$ & \\
\hline
\end{tabular}

BSAi body surface area index; ITT intent-to-treat; SEM standard error of the mean

showed clinical benefit compared with vehicle [14]. However, in the phase $2 \mathrm{~b}$ trial, the primary endpoint was assessed earlier (at Month 1), which may have contributed to the clinical benefit reported in that study [14].

Given that children with EB experience functional impairments that can substantially decrease quality of life [26], and children aged 2 to $<12$ years comprised half $(n=84)$ of the overall study population, planned subgroup analyses were conducted in patients aged 2 to $<12$ years. These analyses demonstrated a trend toward earlier wound closure with SD-101 6\% at Week 2 and Month 1, and the proportion of patients achieving target wound closure numerically favored SD-101 6\% at Month 1 , compared with vehicle. These observations suggest that SD-101 6\% may promote faster wound closure compared with vehicle in patients aged 2 to $<12$ years. Notably, treatment difference (versus vehicle) only reached statistical significance at Week 2 and at Month 1 . Because placebo response would be expected to be highest in patients with mild disease, efficacy was also evaluated in a post hoc analysis of the subgroup of patients with $\geq 5 \%$ BSAi of total body wound burden at baseline. Similar to the subgroup analyses based on age, a positive trend toward wound healing at earlier time points was noted in patients with $\geq 5 \%$ BSAi of total body wound burden at baseline who received SD-101 6\% compared with vehicle. Overall, SD-101 6\% was generally well tolerated, with TEAEs occurring at generally similar frequencies across treatment groups. Two patients who received SD-101 6\% experienced urticaria compared with none in the vehicle arm, suggesting the possibility of a hypersensitivity reaction to SD-101 6\%.

The lack of a statistically significant difference in wound closure between SD-101 and vehicle at Month 3 could be due to a higher-than-expected placebo response rate, which increased over time (manuscript submitted as a companion paper). A high rate of therapeutic success in the vehicle group appears to be a frequent effect in controlled clinical studies of patients with EB [27-31], and may have contributed to the lack of a statistically significant difference in the present study. It is possible that one or more components of the vehicle control cream had therapeutic effects. The vehicle used in the present study contained lanolin oil and cod liver oil, both of which have been shown to promote skin healing [32,33], and therefore could have contributed to the higher-than-expected levels of wound closure observed in the control group. Notably, petroleum, once considered an "inert" moisturizer, was recently shown to robustly modulate antimicrobials and epidermal differentiation [34], further supporting the premise that vehicles 
Table 3 Summary of treatment-emergent adverse events ${ }^{a}$ (safety population)

\begin{tabular}{lll}
\hline $\mathrm{n}(\%)$ & $\begin{array}{l}\text { SD-101 6\% } \\
n=82\end{array}$ & $\begin{array}{l}\text { Vehicle } \\
n=87\end{array}$ \\
\hline Overall Summary & $71(86.6)$ & $61(70.1)$ \\
Any AE & $4(4.9)$ & $8(9.2)$ \\
Any serious AE & 0 & $1(1.1)^{\mathrm{b}}$ \\
Any AE leading to death & $5(6.1)$ & $3(3.4)$ \\
Any AE leading to discontinuation & $9(11.0)$ & $8(9.2)$ \\
AEs Occurring in $\geq 5 \%$ of Patients in Either Treatment Arm & \\
Worsening pruritus & $11(13.4)$ & $3(3.4)$ \\
Nasopharyngitis & $7(8.5)$ & $9(10.3)$ \\
Pyrexia & $6(7.3)$ & $5(5.7)$ \\
Wound infection & $4(4.9)$ & $9(10.3)$ \\
Upper respiratory tract infection & $3(3.7)$ & $9(10.3)$ \\
All skin infection & $1(1.2)$ & $7(8.0)$ \\
Staphylococcal skin infection & & \\
AEs of Special Interest & $15(18.3)$ & $29(33.3)^{d}$ \\
Patients who had skin infection ${ }^{\mathrm{c}}$ & &
\end{tabular}

$A E$ adverse event

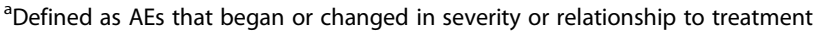
on or after the date of the first dose of study medication

${ }^{b}$ One case of a non-treatment-related death occurred on Day 62 after initiation of treatment owing to cardiac disorders and cardiopulmonary failure 'Patients were considered to have a skin infection if meeting one of the following preferred terms: skin infection, wound infection, staphylococcal skin infection, bacterial skin infection, staphylococcal wound infection, folliculitis, bacterial wound infection, cellulitis, staphylococcal cellulitis, impetigo, infected skin ulcer, postoperative wound infection, or pustular rash

${ }^{\mathrm{d}} P=0.026$ based on Chi-square test

alone may have therapeutic benefit. These observations support the benefits of moisturizing skin care for the management of EB, which have not been formally studied. In addition, daily application of a topical cream to the entire body may have contributed to the improvements observed in the vehicle arm, as the degree of skin care that occurred during the trial may have been greater than the skin care routine normally practiced at home.

Several therapeutic approaches have been evaluated for the treatment of EB. However, most studies were not randomized or controlled, and few have shown any meaningful clinical efficacy [35]. Nonetheless, ongoing research holds promise for new therapeutic strategies. Findings from our study may have important implications for the design of future clinical studies in patients with EB. A placebo run-in period of 3 months' duration preceding randomization should be considered in future EB study designs, allowing the exclusion of "early responders" or those who respond to proper wound care and components of vehicle controls (e.g., lanolin, cod liver oil) prior to randomization to trial treatment. However, this approach may be limited by low enrollment numbers owing to the rarity of $\mathrm{EB}$, as obtaining enough
Table 4 Treatment-related adverse events ${ }^{a}$ (safety population)

\begin{tabular}{|c|c|c|}
\hline n (\%) & $\begin{array}{l}\text { SD-1016\% } \\
n=82\end{array}$ & $\begin{array}{l}\text { Vehicle } \\
n=87\end{array}$ \\
\hline Any treatment-related $\mathrm{AE}$ & $15(18.3)$ & $19(21.8)$ \\
\hline \multicolumn{3}{|c|}{ Treatment-Related AEs Occurring in $\geq 2 \%$ of Patients in Either Treatment Arm } \\
\hline Pruritus (localized or generalized) & $6(7.3)$ & $5(5.7)$ \\
\hline Wound & $2(2.4)$ & $2(2.3)$ \\
\hline Blister & $2(2.4)$ & $2(2.3)$ \\
\hline Urticaria & $2(2.4)$ & 0 \\
\hline Dry skin & $2(2.4)$ & 0 \\
\hline Generalized pruritus & $2(2.4)$ & 0 \\
\hline Staphylococcal skin infection ${ }^{\mathrm{b}}$ & 0 & $4(4.6)$ \\
\hline Skin infection ${ }^{\mathrm{b}}$ & 0 & $3(3.4)$ \\
\hline Dermatitis $^{c}$ & 0 & $2(2.3)$ \\
\hline Maculopapular rash ${ }^{d}$ & 0 & $2(2.3)$ \\
\hline \multicolumn{3}{|c|}{$\begin{array}{l}A E \text { adverse event } \\
{ }^{\mathrm{a}} \text { AEs were deemed unrelated, possibly, probably, or definitely related to } \\
\text { treatment by the investigator } \\
{ }^{\mathrm{b}} \text { Staphylococcal skin infection was confirmed with either microbiology sample } \\
\text { or antibiotic treatment, and skin infection included all unspecified } \\
\text { skin infection } \\
{ }^{\mathrm{C} D e s c r i b e d ~ a s ~ d e r m a t i t i s ~ w i t h o u t ~ p r u r i t u s ~ o r ~ p a i n ~}(n=1) \text { and lumber area skin } \\
\text { inflammation }(n=1) \\
{ }^{d} \text { Patients with maculopapular rash are different from those with dermatitis }\end{array}$} \\
\hline
\end{tabular}

patients to attain sufficient power to detect efficacy is challenging even with a global enrollment strategy. In addition, given that patients with EB have multiple wounds that fluctuate and recur, future studies should include whole patient assessments, such as the validated EBDASI, iScorEB, and QOLEB scores. The possibility that our study was confounded by a high rate of vehicle response is supported by the post hoc subgroup analysis in patients with BSAi of total wound burden $\geq 5 \%$. When patients with the mildest disease $(\mathrm{BSAi}<5 \%)$ were removed from the analysis, numerically greater differences between SD-101 6\% and vehicle were observed. Similarly, patients aged $<2$ years who were treated with vehicle showed a numerically higher response than other age groups (manuscript submitted as a companion paper); when this age group was excluded, greater improvements with SD-101 6\% compared with vehicle were apparent in patients aged 2 to $<12$ years of age.

\section{Conclusions}

ESSENCE included a broad representation of EB subtypes and is the largest randomized, controlled clinical trial of an investigational drug in patients with EB completed to date. In this study, no significant differences between SD-101 6\% and vehicle were observed for the primary efficacy endpoints of time to complete target wound closure or the proportion of patients with complete target wound closure within 3 months. A positive trend toward faster wound healing with SD-101 6\% 
at earlier time points (within 1 month) was noted for subgroups of patients between 2 and $<12$ years of age and those with $\geq 5 \%$ BSAi of total body wound burden at baseline. SD-101 6\% was generally well-tolerated with a safety profile similar to that of vehicle.

\section{Supplementary information}

Supplementary information accompanies this paper at https://doi.org/10. 1186/s13023-020-01419-3.

Additional file 1:. ESSENCE individual patient data. Contains individual de-identified participant data regarding the target wound from the current study.

Additional file 2:. ESSENCE study site information. Presents study investigators, country, and patient numbers for all study sites.

\section{Abbreviation}

AE: adverse event; BSAi: body surface area index; $\mathrm{Cl}$ : confidence interval; D: day; EB: Epidermolysis bullosa; FLACC: Face, Legs, Activity, Cry, Consolability scale; ITT: intent-to-treat; M: month; NC: not calculated; RDEB: recessive dystrophic epidermolysis bullosa; SD: standard deviation; SE: standard error; SEM: standard error of the mean

\section{Acknowledgments}

The authors thank the patients and their families, as well as the study investigators (see Additional file 2). Third-party medical writing assistance was provided by Sally-Anne Mitchell, PhD, Lynn Brown, PhD, and Lei Bai, PhD, of ApotheCom (Yardley, PA) and was supported by Amicus Therapeutics, Inc.

\section{Funding information}

The study was supported by Amicus Therapeutics, Inc. This study was designed, managed, and analyzed jointly by authors employed by Amicus Therapeutics, Inc., and external authors who were not paid for their work

\section{Authors' contributions}

$A S P, A R$, HL, and JAB participated in study design; ASP, JB, MN, CB, DFM, WL, and $\mathrm{HL}$ assisted in the acquisition of data; $\mathrm{ASP}, \mathrm{HL}$, and $\mathrm{EK}$ analyzed data; ASP, $H L$, and JAB interpreted the data; $H L$ drafted the manuscript. All authors critically revised the manuscript; all authors gave final approval of the submitted version and agreed to be accountable for all aspects of the work in ensuring that questions related to the accuracy or integrity of any part of the work are appropriately investigated and resolved.

\section{Availability of data and materials}

Individual de-identified participant data regarding the target wound from the current study are published as supplemental material.

\section{Ethics approval and consent to participate}

The study was conducted in accordance with ethical principles founded in the Declaration of Helsinki and in compliance with the protocol, International Conference on Harmonisation Good Clinical Practice, and applicable local legal and regulatory requirements. An Institutional Review Board/Independent Ethics Committee reviewed and approved the study design and protocol at each study site, and signed informed consent/assent was obtained from each patient (or legal guardian if under 18 years of age) prior to entering the study.

\section{Consent for publication}

Not applicable.

\section{Competing interests}

ASP has served as an investigator (uncompensated) for Amicus, Castle Creek Pharmaceuticals, Lenus Therapeutics, and Shire and as a consultant with honorarium for Castle Creek Pharmaceuticals, Lenus Therapeutics, and MEDACorp. JB has served as an investigator for Amicus, Galderma, Valeant, Patagonia, and Regeneron and as a speaker for Medimetriks and Promius. MN has received honoraria from Meda/Mylan, Medis, La Roche-Posay/L'Oreal,
Uriage, Takeda, Novartis, Janssen, and Amicus. CB has served on advisory boards for Sanofi, as a consultant for Expanscience, as an investigator (uncompensated) for Amicus, as a speaker for La Roche-Posay and Expanscience, and has received honoraria from Celgene and Novartis. DFM has served as a consultant and investigator for Amicus, Amryt, Castle Creek Pharmaceuticals, and Shire and has conducted her own investigator-initiated randomized controlled trials in recessive dystrophic epidermolysis bullosa and epidermolysis bullosa simplex. EK is a paid consultant of Amicus. AR, HL, and JAB are employees of and hold stock in Amicus.

\section{Author details}

${ }^{1}$ Northwestern University Feinberg School of Medicine, Chicago, IL, USA.

${ }^{2}$ Texas Dermatology \& Laser Specialists, San Antonio, TX, USA. ${ }^{3}$ Clinical Center of Serbia, Department of Dermatology, University of Belgrade, Belgrade, Serbia. ${ }^{4}$ EB Reference Centre, Department of Dermatology, University Hospital Necker Enfants Malades, Paris, France. ${ }^{5}$ University of New South Wales, Sydney, NSW, Australia. ${ }^{6}$ Scioderm-An Amicus Therapeutics Company, Durham, NC, USA. ${ }^{7}$ Amicus Therapeutics, Inc, Cranbury, NJ, USA.

Received: 20 February 2020 Accepted: 25 May 2020

Published online: 23 June 2020

\section{References}

1. El Hachem M, Zambruno G, Bourdon-Lanoy E, Ciasulli A, Buisson C, HadjRabia S, et al. Multicentre consensus recommendations for skin care in inherited epidermolysis bullosa. Orphanet J Rare Dis. 2014;9:76.

2. Fine JD. Inherited epidermolysis bullosa. Orphanet J Rare Dis. 2010;5:12.

3. Ma JE, Hand JL. What's new with common genetic skin disorders? Minerva Pediatr. 2017.

4. Brandling-Bennett HA, Morel KD. Common wound colonizers in patients with epidermolysis bullosa. Pediatr Dermatol. 2010;27(1):25-8.

5. Tabolli S, Pagliarello C, Uras C, Di Pietro C, Zambruno G, Castiglia D, et al. Family burden in epidermolysis bullosa is high independent of disease type/subtype. Acta Derm Venereol. 2010;90(6):607-11.

6. Tabor A, Pergolizzi JV Jr, Marti G, Harmon J, Cohen B, Lequang JA. Raising awareness among healthcare providers about epidermolysis bullosa and advancing toward a cure. J Clin Aesthet Dermatol. 2017:10(5):36-48.

7. Pagliarello C, Tabolli S. Factors affecting quality of life in epidermolysis bullosa. Expert review of pharmacoeconomics \& outcomes research. 2010; 10(3):329-38.

8. Jain SV, Murrell DF. Psychosocial impact of inherited and autoimmune blistering diseases. Int J Women's Dermatol. 2018;4(1):49-53.

9. Fine JD. Epidemiology of inherited epidermolysis bullosa based on incidence and prevalence estimates from the national epidermolysis bullosa registry. JAMA Dermatol. 2016;152(11):1231-8.

10. Debra UK. What is EB. https://wwwdebra.org.uk/what-is-eb/what-is-eb. Accessed 2 June 2020

11. Fine JD, Bruckner-Tuderman L, Eady RA, Bauer EA, Bauer JW, Has C, et al. Inherited epidermolysis bullosa: updated recommendations on diagnosis and classification. J Am Acad Dermatol. 2014;70(6):1103-26.

12. Uitto J, Bruckner-Tuderman L, Christiano AM, McGrath JA, Has C, South AP, et al. Progress toward treatment and cure of epidermolysis bullosa: summary of the DEBRA International research symposium EB2015. J Invest Dermatol. 2016;136(2):352-8

13. Goldschneider KR, Good J, Harrop E, Liossi C, Lynch-Jordan A, Martinez AE, et al. Pain care for patients with epidermolysis bullosa: best care practice guidelines. BMC Med. 2014;12:178.

14. Paller A, Browning J, Aslam R, Bayliss SJ, Lugo-Somolinos A, Sidbury R, et al. Twelve-month efficacy and safety findings of SD-101 cream from an openlabel extension study on reducing body surface area coverage of lesional skin in patients with epidermolysis bullosa. 42nd Annual Meeting of the Society for Pediatric Diseases; Minneapolis, MN 2016

15. Aggarwal SR. A survey of breakthrough therapy designations. Nat Biotechnol. 2014:32(4):323-30.

16. Has C, Bauer JW, Bodemer C, Bolling MC, Bruckner-Tuderman L, Diem A et al. Consensus reclassification of inherited epidermolysis bullosa and other disorders with skin fragility. Br J Dermatol. 2020. https://doi.org/10.1111/bjd. 18921 [Epub ahead of print].

17. Yenamandra VK, Moss C, Sreenivas V, Khan M, Sivasubbu S, Sharma VK, et al. Development of a clinical diagnostic matrix for characterizing inherited epidermolysis bullosa. Br J Dermatol. 2017;176(6):1624-32. 
18. Morris V, Murphy LM, Rosenberg M, Rosenberg L, Holzer CE 3rd, Meyer WJ 3rd. Itch assessment scale for the pediatric burn survivor. J Burn Care Res. 2012;33(3):419-24.

19. Merkel SI, Voepel-Lewis T, Shayevitz JR, Malviya S. The FLACC: a behavioral scale for scoring postoperative pain in young children. Pediatr Nurs. 1997; 23(3):293-7.

20. Wong DL, Baker CM. Smiling faces as anchor for pain intensity scales. Pain. 2001;89(2-3):295-300.

21. Davila-Seijo P, Hernandez-Martin A, Morcillo-Makow E, de Lucas R Dominguez $E$, Romero $N$, et al. Prioritization of therapy uncertainties in dystrophic Epidermolysis Bullosa: where should research direct to? An example of priority setting partnership in very rare disorders. Orphanet J Rare Dis. 2013;8:61

22. Ozcelik B, Kartal M, Orhan I. Cytotoxicity, antiviral and antimicrobial activities of alkaloids, flavonoids, and phenolic acids. Pharm Biol. 2011:49(4):396-402.

23. Araujo LU, Grabe-Guimaraes A, Mosqueira VC, Carneiro CM, Silva-Barcellos NM. Profile of wound healing process induced by allantoin. Acta Cir Bras. 2010;25(5):460-6.

24. Robinson W. Stimulation of healing in non-healing wounds: by allantoin occurring in maggot secretions and of wide biological distribution. J Bone Joint Surg. 1935;17:267-71.

25. Lo V, Lara-Corrales I, Stuparich A, Pope E. Amniotic membrane grafting in patients with epidermolysis bullosa with chronic wounds. J Am Acad Dermatol. 2010;62(6):1038-44.

26. Eismann EA, Lucky AW, Cornwall R. Hand function and quality of life in children with epidermolysis bullosa. Pediatr Dermatol. 2014;31(2):176-82.

27. Chiaverini C, Roger C, Fontas E, Bourrat E, Bourdon-Lanoy E, Labreze C, et al. Oral epigallocatechin-3-gallate for treatment of dystrophic epidermolysis bullosa: a multicentre, randomized, crossover, double-blind, placebocontrolled clinical trial. Orphanet J Rare Dis. 2016;11:31.

28. Wally V, Kitzmueller S, Lagler F, Moder A, Hitzl W, Wolkersdorfer M, et al. Topical diacerein for epidermolysis bullosa: a randomized controlled pilot study. Orphanet J Rare Dis. 2013;8:69.

29. Lara-Corrales I, Parkin PC, Stephens D, Hamilton J, Koren G, Weinstein M, et al. The efficacy of trimethoprim in wound healing of patients with epidermolysis bullosa: a feasibility trial. J Am Acad Dermatol. 2012;66(2):264-70.

30. Weiner M, Stein A, Cash S, de Leoz J, Fine JD. Tetracycline and epidermolysis bullosa simplex: a double-blind, placebo-controlled, crossover randomized clinical trial. Br J Dermatol. 2004;150(3):613-4

31. Caldwell-Brown D, Stern RS, Lin AN, Carter DM. Lack of efficacy of phenytoin in recessive dystrophic epidermolysis bullosa. Epidermolysis Bullosa study group. N Engl J Med. 1992;327(3):163-7.

32. Chvapil M, Gaines JA, Gilman T. Lanolin and epidermal growth factor in healing of partial-thickness pig wounds. J Burn Care Rehabil. 1988;9(3):279-84.

33. Terkelsen LH, Eskild-Jensen A, Kjeldsen H, Barker JH, Hjortdal VE. Topical application of cod liver oil ointment accelerates wound healing: an experimental study in wounds in the ears of hairless mice. Scand J Plast Reconstr Surg Hand Surg. 2000;34(1):15-20.

34. Czarnowicki T, Malajian D, Khattri S, Correa da Rosa J, Dutt R, Finney R, et al. Petrolatum: Barrier repair and antimicrobial responses underlying this "inert" moisturizer. J Allergy Clin Immunol. 2016;137(4):1091-102.e7.

35. Langan SM, Williams HC. A systematic review of randomized controlled trials of treatments for inherited forms of epidermolysis bullosa. Clin Exp Dermatol. 2009;34(1):20-5.

\section{Publisher's Note}

Springer Nature remains neutral with regard to jurisdictional claims in published maps and institutional affiliations.

Ready to submit your research? Choose BMC and benefit from:
- fast, convenient online submission
- thorough peer review by experienced researchers in your field
- rapid publication on acceptance
- support for research data, including large and complex data types
- gold Open Access which fosters wider collaboration and increased citations
- maximum visibility for your research: over 100M website views per year
At BMC, research is always in progress.
Learn more biomedcentral.com/submissions

